\title{
Adjacent-segment disease after L3-4 posterior lumbar interbody fusion: does L3-4 fusion have cranial adjacent-segment degeneration similar to that after L4-5 fusion?
}

\author{
Hiroyuki Aono, MD, PhD, ${ }^{1}$ Shota Takenaka, MD, PhD, ${ }^{2}$ Hidekazu Tobimatsu, MD, ${ }^{3}$ \\ Yukitaka Nagamoto, MD, PhD, ${ }^{4}$ Masayuki Furuya, MD, PhD, ${ }^{1}$ Tomoya Yamashita, MD, ${ }^{1}$ \\ Hiroyuki Ishiguro, MD, $\mathrm{PhD},{ }^{1}$ and Motoki Iwasaki, MD, $\mathrm{PhD}^{4}$ \\ ${ }^{1}$ National Hospital Organization, Osaka National Hospital; ${ }^{2}$ Osaka University Graduate School of Medicine; 3Bell Land General \\ Hospital; and ${ }^{4}$ Osaka Rosai Hospital, Osaka, Japan
}

\begin{abstract}
OBJECTIVE Posterior lumbar interbody fusion (PLIF) is a widely accepted procedure for degenerative lumbar diseases, and there have been many reports concerning adjacent-segment disease (ASD) after PLIF. In the reports of ASD in which the fusion level was limited to 1 segment, all reports describe ASD of the L3-4 segment after L4-5 PLIF. On the basis of these reports, it is thought that ASD mainly occurs at the cranial segment. However, no report has covered ASD after L3-4 PLIF. Therefore, the authors investigated ASD after L3-4 PLIF.

METHODS In conducting a retrospective case series analysis, the authors reviewed a surgical database providing details of all spine operations performed between 2006 and 2017 at a single institution. During that period, PLIF was performed to treat 632 consecutive patients with degenerative lumbar diseases. Of these patients, 71 were treated with L3-4 PLIF alone, and 67 who were monitored for at least 2 years (mean 5.8 years; follow-up rate 94\%) after surgery were enrolled in this study. Radiological ASD (R-ASD), symptomatic ASD (S-ASD), and operative ASD (O-ASD) were evaluated. These types of ASD were defined as follows: R-ASD refers to radiological degeneration adjacent to the fusion segment as shown on plain radiographs; S-ASD is a symptomatic condition due to neurological deterioration at the adjacent-segment degeneration; and O-ASD refers to S-ASD requiring revision surgery.
\end{abstract}

RESULTS All patients had initial improvement of neurological symptoms after primary PLIF. R-ASD was observed in $32(48 \%)$ of 67 patients. It occurred at the cranial segment in 12 patients and at the caudal segment in 24; R-ASD at both adjacent segments was observed in 4 patients. Thus, the occurrence of R-ASD was more significant in the caudal segment than in the cranial segment. S-ASD was observed in 10 patients (15\%), occurring at the cranial segment in 3 patients and at the caudal segment in 7. O-ASD was observed in 6 patients (9\%): at the cranial segment in 1 patient and at the caudal segment in 5 . Thus, the rate of involvement of the caudal segment was $67 \%$ in R-ASD, $70 \%$ in S-ASD, and $83 \%$ in O-ASD.

CONCLUSIONS The incidences of R-ASD, S-ASD, and O-ASD were 48\%, 15\%, and 9\%, respectively, after L3-4 PLIF for degenerative lumbar diseases. In contrast to ASD after L4-5 PLIF, ASD after L3-4 PLIF was more frequently observed at the caudal segment than at the cranial segment. In follow-up for patients with L3-4 PLIF, surgeons should pay attention to ASD in the caudal segment.

https://thejns.org/doi/abs/10.3171/2020.3.SPINE20122

KEYWORDS posterior lumbar interbody fusion; PLIF; adjacent-segment disease; L3-4; late complication

$\mathrm{P}$ OSTERIOR lumbar interbody fusion (PLIF) has become a widely accepted procedure for degenerative lumbar diseases because of the development of spinal instrumentation, including interbody cages. PLIF has a number of advantages over other forms of fusion surgery, including the circumferential decompression of neural elements, a high fusion rate, good correction, and maintenance of lumbar alignment. ${ }^{1,2}$

Many reports are available about adjacent-segment disease (ASD) after PLIF, and ASD after fusion surgery is 


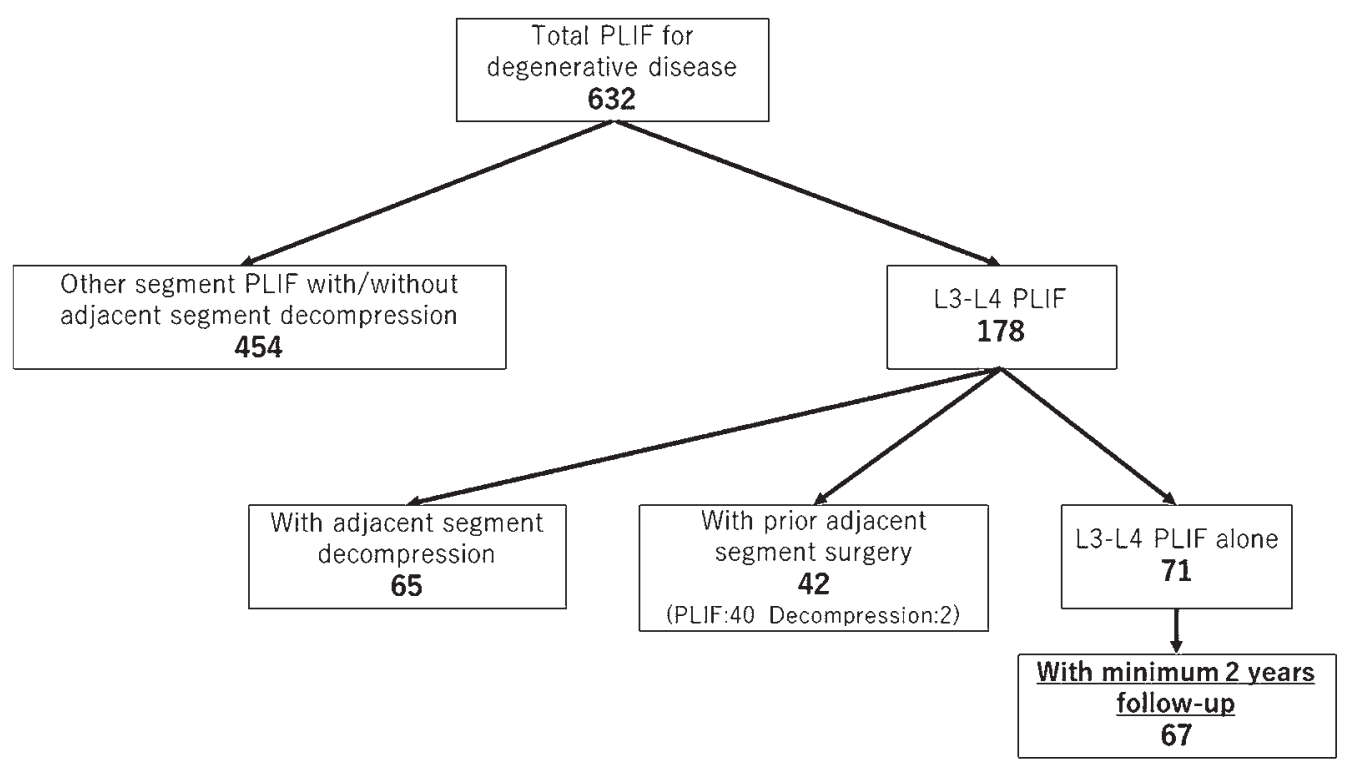

FIG. 1. Flow diagram with number of patients included/excluded. Of 632 patients treated with PLIF, only 71 (11\%) were treated with L3-4 PLIF alone (without additional adjacent-segment decompression), and 67 were monitored for at least 2 years.

frequently observed at a cranial segment. ${ }^{3,4}$ In reports of ASD in which the fusion level is limited to 1 segment, ASD is described as affecting the L3-4 segment after L4-5 fusion surgery, ${ }^{5-9}$ because spondylolisthesis at L4 is the most common indication for fusion surgery. There are no reports focusing on ASD after L3-4 fusion surgery.

We therefore hypothesized that ASD after L3-4 PLIF is different from ASD after L4-5 PLIF, because the caudal L5-S1 segment is fixed by rigid lumbosacral ligaments in L4-5 PLIF. Moreover, it is assumed that there may be a feature of ASD after L3-4 PLIF. We conducted a study to evaluate ASD after L3-4 PLIF.

\section{Methods}

\section{Patient Population}

We retrospectively reviewed the surgical database providing prospectively collected details of all spine operations performed between June 2006 and July 2017 at our institution. During that period, we performed PLIF either with or without additional adjacent-segment decompression to treat 632 consecutive patients with degenerative lumbar diseases. Of these patients, 71 were treated with L3-4 PLIF alone (without additional adjacent-segment decompression), and 67 of the 71 who were monitored for at least 2 years after surgery were enrolled in our retrospective study (Fig. 1). The follow-up rate was $94 \%$, and the mean follow-up period was 5.8 years (range 2-13 years). We excluded patients from this study who had prior surgery adjacent to the L3-4 segment, trauma, and infection. The study protocol was approved by the institutional review board of our hospital.

\section{Surgical Indications and Procedures}

All patients were treated for severe pain in their lower extremities with or without low-back pain-receiving con- servative treatment preoperatively that did not sufficiently relieve their pain. The indications for PLIF in patients with spondylolisthesis were slippage of $\geq 3 \mathrm{~mm}$ and/or posterior opening of the disc space of $\geq 5^{\circ}$ on lateral flexion radiographs. PLIF was also performed to treat foraminal stenosis lesions.

\section{Surgical Techniques}

All PLIF procedures were performed using the same technique described previously. ${ }^{10,11}$ In summary, our techniques consisted of bilateral total facetectomy; subtotal discectomy; a large amount of local bone graft, which means positioning residual morselized bone into the anterior portion of the interbody space and at least 2 strut bone blocks with 2 box-type polyetheretherketone cages; and pedicle screw instrumentation (Fig. 2).

\section{Outcome Measures}

Complete medical records of all patients were available for review. Clinical and radiological assessments were performed for all patients before surgery, at 6 and 12 months after surgery, and annually thereafter. Radiographic measurements were performed in blinded fashion by 3 independent spine surgeons.

ASD was defined as follows: radiological ASD (R-ASD) on plain radiographs as reported by Nakashima et al. ${ }^{12}$ Conditions in which there was slippage progression of $>3 \mathrm{~mm}$, a posterior opening of $>5^{\circ}$, and a narrowing of disc height of $>3 \mathrm{~mm}$ in comparison with preoperative flexion-extension lateral radiographs were considered R-ASD. Symptomatic ASD (S-ASD) was diagnosed when clinical symptoms such as leg pain or intermittent claudication appeared during the follow-up period and the responsible lesion was confirmed by MRI to be in a segment adjacent to the PLIF level. Operative ASD (O-ASD) was 


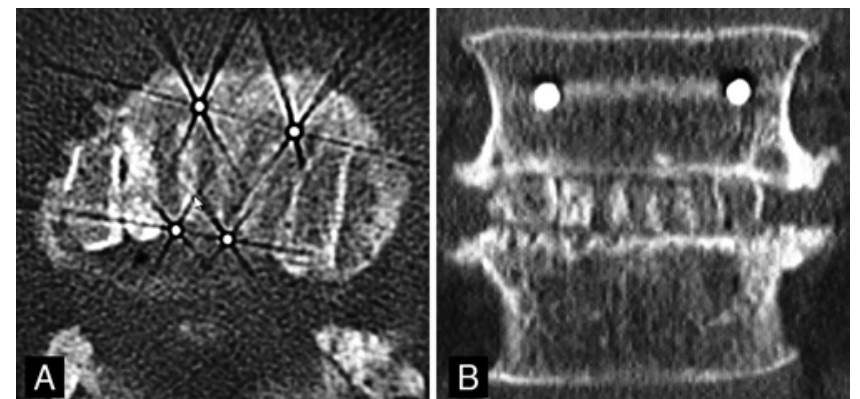

FIG. 2. Axial (A) and coronal (B) CT images of bone graft. Residual morselized bone was placed in the anterior portions of interbody spaces, with 2 cages sandwiched between a minimum of 2 autologous strut blocks.

defined as a symptomatic condition in which additional surgery was required to treat neurological deterioration of the adjacent degenerating segment. We also evaluated how long it took for ASD to be evident.

Standing and flexion-extension lateral radiographs of the lumbar spine were obtained preoperatively and just after surgery. Lumbar lordosis, defined as the angle between the superior endplate of L1 and the superior endplate of S1 in the standing neutral position, was measured to assess sagittal alignment of the lumbar spine. Segmental lordosis, defined as the angle between the superior endplate of L3 and the inferior endplate of L4 was also measured.

Fusion status was assessed 1 year after surgery. All patients underwent postoperative multiplanar reconstruction CT scans at 1 year after surgery. Solid fusion was defined as demonstrated osseous continuity between the graft bone and the vertebrae, with no obvious clear zone between the graft bone/cages and vertebrae on CT, without motion at the fused levels on flexion-extension radiographs.

We also evaluated preoperative adjacent-disc degeneration according to the Pfirrmann classification ${ }^{13}$ and analyzed the difference of grade at each adjacent segment, grouping them as R-ASD, S-ASD, or O-ASD.

\section{Statistical Analysis}

We used SPSS statistical software (version 21.0; IBM Corp.) for all analyses; statistical significance was set at $\mathrm{p}$ $<0.05$. The Fisher exact test was used to compare group differences for categorical variables.

\section{Results}

Our study group consisted of 24 men and 43 women with a mean age of 69.7 years (range $43-83$ years). Of these, 62 had degenerative spondylolisthesis (Meyerding grade I, 49; grade II, 10), 4 had foraminal stenosis, and 1 had isthmic spondylolisthesis. All patients had initial improvement of neurological symptoms after primary PLIF.

With regard to fusion status, solid fusion was achieved in 66 patients by the 1-year examination, and the remaining patient had solid fusion by the 1.5 -year examination.

R-ASD was observed in $32(48 \%)$ of 67 patients (Table 1). Concerning the type of ASD, slippage progression was observed in 10 patients (cranial in 3, caudal in 7) and a
TABLE 1. ASD after L3-4 PLIF ( $\mathrm{N}=67$ )

\begin{tabular}{cccccc}
\hline \multirow{2}{*}{$\begin{array}{c}\text { Type of } \\
\text { ASD }\end{array}$} & $\begin{array}{c}\text { No. of } \\
\text { Pts }^{*}\end{array}$ & \multicolumn{3}{c}{ Spine Segment } & \\
\cline { 3 - 5 } R-ASD & 32 & 8 & 20 & 4 & 0.031 \\
\hline S-ASD & 10 & 3 & 7 & 0 & 0.481 \\
\hline O-ASD & 6 & 1 & 5 & 0 & 0.391 \\
\hline
\end{tabular}

Pts = patients.

* Of the 67 patients, 32 had ASD.

posterior opening was observed in 6 patients (cranial in 2, caudal in 4). Narrowing of the disc height was most frequently observed in 20 patients (cranial in 7, caudal in 13). ASD at both adjacent segments was observed in 4 patients (Table 2). R-ASD was observed at 36 months (range 12-72 months) after initial PLIF. Thus, R-ASD was significantly more often observed in a caudal segment than in a cranial segment (caudal in 24, cranial in 12; $\mathrm{p}=0.031$ ).

S-ASD was observed in 10 patients $(15 \%$ - cranial in 3 , caudal in 7; $p=0.481$ ). The causes of S-ASD were stenosis of a cranial segment in 3 patients (with slippage in 2 patients, without slippage in 1 patient); stenosis of a caudal segment in 7 (with slippage in 4, without slippage in 2, foraminal stenosis in 1; Fig. 3, Table 1). S-ASD appeared at a mean of 44 months (range 16-78 months) after initial PLIF.

O-ASD was observed in 6 patients (9\%). Only 1 patient had the condition at a cranial segment, whereas 5 patients had it at a caudal segment (Table 1). The mean amount of time between primary surgery and revision surgery was 56 months (range 20-90 months). Concerning pathologies of O-ASD, degenerative spondylolisthesis was observed in 4 patients (Fig. 4) and spinal stenosis in 2 patients.

Among the patients with ASD, the rate of involvement of a caudal segment was $67 \%$ for R-ASD, $70 \%$ for S-ASD, and $83 \%$ for O-ASD. Thus, ASD was most frequently observed at a caudal segment.

Concerning sagittal alignment, lumbar lordosis increased from $27.9 \pm 12.1$ before surgery to $34.4 \pm 12.2$ after surgery, and segmental lordosis increased from 1.7 \pm 5.1 before surgery to $3.5 \pm 3.7$ after surgery. Statistical analysis revealed that patients with S-ASD had significantly poor segmental lordosis both before and after surgery, whereas patients with O-ASD had significantly poor segmental lordosis only before surgery (Table 3 ).

Preoperative disc degeneration evaluated by the Pfirr-

TABLE 2. R-ASD after L3-4 PLIF $(\mathrm{n}=32)$

\begin{tabular}{lcc}
\hline \multirow{2}{*}{ Parameter } & \multicolumn{2}{c}{ Spine Segment } \\
\cline { 2 - 3 } & Cranial $(\mathrm{n})$ & Caudal $(\mathrm{n})$ \\
\hline Slippage progression & 3 & 7 \\
\hline Posterior opening & 2 & 4 \\
\hline Narrowing of disc height & 7 & 13 \\
\hline Total $^{*}$ & 12 & 24 \\
\hline
\end{tabular}

${ }^{*}$ ASD at both adjacent segments was observed in 4 patients. 


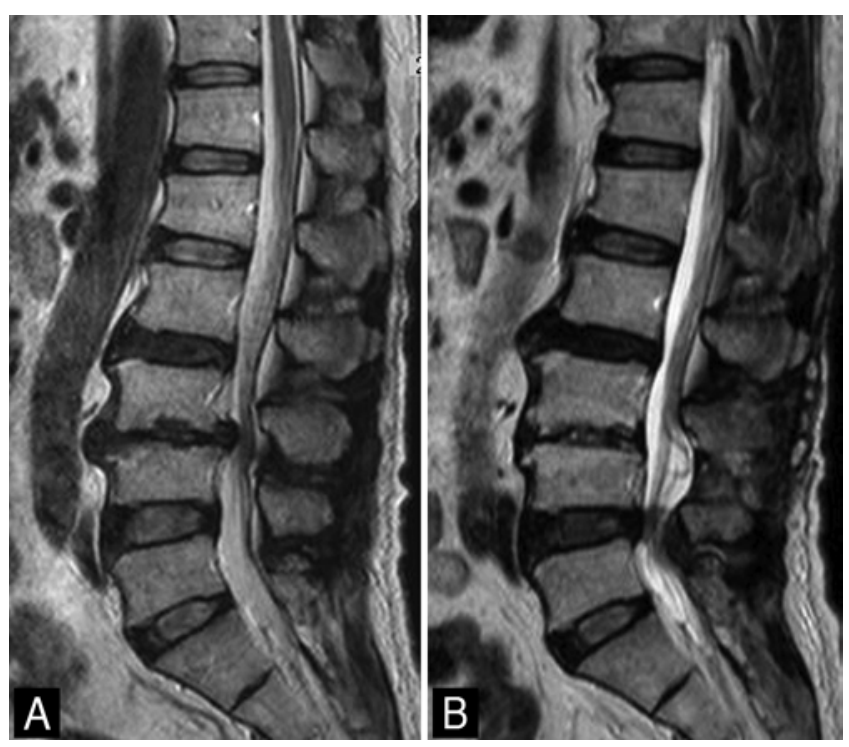

FIG. 3. Transitional change shown by sagittal MRI obtained in a 70-yearold man with L3 spondylolisthesis. A: Before L3-4 PLIF, there was no stenosis at the L4-5 segment. B: Four and one-half years after surgery, neurological claudication appeared and stenosis at the L4-5 segment is clear. The patient has been treated conservatively.

mann classification was grade II in 1 patient, grade III in 8 , grade IV in 54, and grade $\mathrm{V}$ in 4 patients at the L2-3 segment, and it was grade III in 2 , grade IV in 48 , and grade $\mathrm{V}$ in 17 patients at the L4-5 segment. There was no significant difference in Pfirrmann grades of any type of ASD in either adjacent segment (Table 4).

\section{Discussion}

Since Cloward ${ }^{14}$ first reported PLIF for lumbar disc
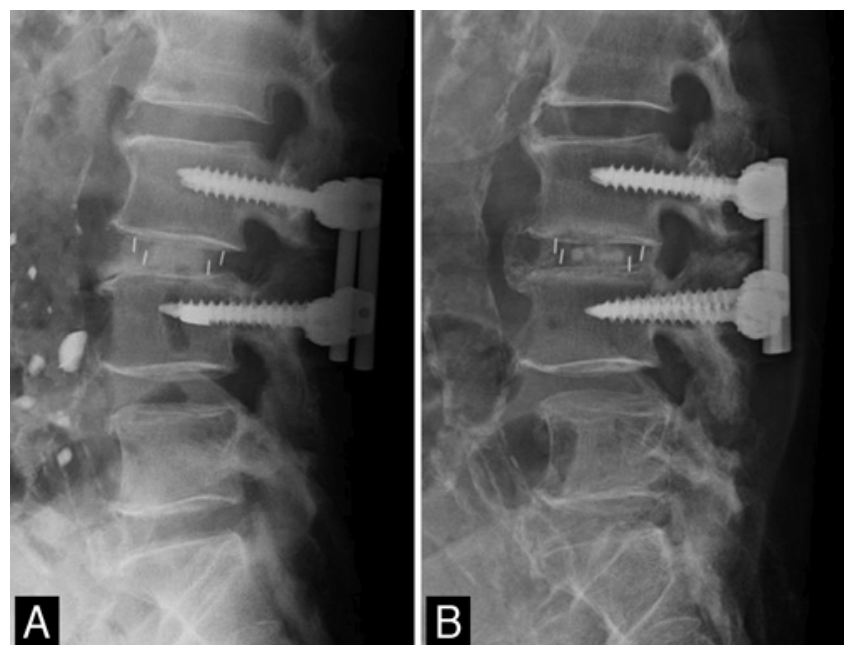

FIG. 4. Lateral radiographs obtained in a 72-year-old man with $L 3$ spondylolisthesis, showing changes over time. A: Just after surgery. B: Five years after surgery, neurological claudication became severe and $\mathrm{L} 4$ spondylolisthesis is obvious. The patient was treated with additional L4-5 PLIF.
TABLE 3. Relation between ASD and sagittal alignment after L3-4 PLIF ( $\mathrm{N}=67)$

\begin{tabular}{|c|c|c|c|c|c|}
\hline \multirow[b]{2}{*}{ Type of ASD } & \multicolumn{2}{|c|}{ Parameter } & \multicolumn{2}{|c|}{ ASD } & \multirow[b]{2}{*}{$p$ Value } \\
\hline & Alignment & Time Point & Yes & No & \\
\hline \multirow{4}{*}{$\begin{array}{l}\text { R-ASD (degree)- } \\
\text { with, } 32 \text { pts; } \\
\text { without, } 35 \text { pts }\end{array}$} & \multirow{2}{*}{ LL } & Preop & 27.7 & 28.1 & 0.903 \\
\hline & & Postop & 33.5 & 35.2 & 0.570 \\
\hline & \multirow{2}{*}{ SL } & Preop & 1.4 & 2.1 & 0.555 \\
\hline & & Postop & 3.4 & 3.6 & 0.838 \\
\hline \multirow{4}{*}{$\begin{array}{l}\text { S-ASD—with, } 10 \\
\text { pts; without, } 57 \text { pts }\end{array}$} & \multirow{2}{*}{ LL } & Preop & 22.3 & 28.9 & 0.114 \\
\hline & & Postop & 27.6 & 35.6 & 0.056 \\
\hline & \multirow{2}{*}{$S L$} & Preop & -3.1 & 2.6 & 0.001 \\
\hline & & Postop & 0.9 & 4.0 & 0.002 \\
\hline \multirow{4}{*}{$\begin{array}{l}\text { O-ASD_with, } 6 \\
\text { pts; without, } 61 \text { pts }\end{array}$} & \multirow{2}{*}{ LL } & Preop & 25.8 & 28.1 & 0.664 \\
\hline & & Postop & 32.0 & 34.6 & 0.615 \\
\hline & \multirow{2}{*}{ SL } & Preop & -2.6 & 2.2 & 0.024 \\
\hline & & Postop & 1.2 & 3.8 & 0.108 \\
\hline
\end{tabular}

$\mathrm{LL}=$ lumbar lordosis; $\mathrm{SL}=$ segmental lordosis.

herniation, the procedure has been supplemented by posterior instrumentation and intervertebral disc spacers. It is indicated for various spine disorders, especially for segmental spine instability. However, ASD is considered to be one of the common late postoperative complications after PLIF, as noted in a large number of reports.

TABLE 4. Relation between ASD and the Pfirrmann grade after L3-4 PLIF ( $\mathrm{N}=67$ )

\begin{tabular}{|c|c|c|c|c|c|}
\hline \multirow[b]{2}{*}{ Type of ASD } & \multirow[b]{2}{*}{ Segment } & \multirow[b]{2}{*}{ Grade } & \multicolumn{2}{|c|}{ ASD } & \multirow{2}{*}{$\begin{array}{c}p \\
\text { Value }\end{array}$} \\
\hline & & & No. With & No. Without & \\
\hline \multirow{7}{*}{$\begin{array}{l}\text { R-ASD (de- } \\
\text { gree)-with, } \\
32 \text { pts; without, } \\
35 \text { pts }\end{array}$} & \multirow{4}{*}{ L2-3 } & II & 1 & 0 & \multirow{4}{*}{0.180} \\
\hline & & III & 4 & 4 & \\
\hline & & IV & 27 & 27 & \\
\hline & & V & 0 & 4 & \\
\hline & \multirow{3}{*}{ L4-5 } & III & 1 & 1 & \multirow{3}{*}{0.789} \\
\hline & & IV & 24 & 24 & \\
\hline & & V & 7 & 10 & \\
\hline \multirow{7}{*}{$\begin{array}{l}\text { S-ASD_with, } \\
10 \text { pts; without, } \\
57 \text { pts }\end{array}$} & \multirow{4}{*}{ L2-3 } & II & 0 & 1 & \multirow{4}{*}{1.000} \\
\hline & & III & 1 & 7 & \\
\hline & & IV & 9 & 45 & \\
\hline & & V & 0 & 4 & \\
\hline & \multirow{3}{*}{ L4-5 } & III & 0 & 2 & \multirow{3}{*}{0.793} \\
\hline & & IV & 7 & 41 & \\
\hline & & V & 3 & 14 & \\
\hline \multirow{7}{*}{$\begin{array}{l}\text { O-ASD_with, } \\
6 \text { pts; without, } \\
61 \text { pts }\end{array}$} & \multirow{4}{*}{ L2-3 } & $\|$ & 0 & 1 & \multirow{4}{*}{0.741} \\
\hline & & III & 1 & 7 & \\
\hline & & IV & 5 & 49 & \\
\hline & & V & 0 & 4 & \\
\hline & \multirow{3}{*}{ L4-5 } & III & 0 & 2 & \multirow{3}{*}{0.708} \\
\hline & & IV & 4 & 44 & \\
\hline & & V & 2 & 15 & \\
\hline
\end{tabular}


Okuda et al. ${ }^{9}$ reported that no patients developed deterioration of a caudal segment (L5-S1) in their case series of 87 patients who underwent L4-5 PLIF. Nakai et al. ${ }^{3}$ reported that they often observed ASD at a cranial adjacent segment after PLIF after a mean of 8.6 years of follow-up. Kaito et al. ${ }^{7}$ also reported that none of their 85 patients underwent surgery at the L5-S1 level after L4-5 PLIF. Thus, it has been thought that ASD often occurs after PLIF at a cranial adjacent segment. Okuda et al. ${ }^{15}$ also reported that O-ASD was mainly observed at a cranial segment (77\%), followed in frequency by a caudal segment (13\%) and then by both segments $(10 \%)$. Their study involved a minimum 10 years' follow-up of patients who underwent L4-5 PLIF for L4 degenerative spondylolisthesis. Therefore, all reports of studies of ASD after PLIF, which restricted fusion level, described L3-4 ASD occurring after L4-5 PLIF, 5-9 and no study focused on ASD occurring after L3-4 PLIF.

There are several factors why the L4-5 segment is likely to degenerate after L3-4 PLIF. One factor is a difference in anatomical structure. The L5-S1 segment is fixed by rigid lumbosacral ligaments. Therefore, the L3-4 segment, because it is not thus fixed, can more easily degenerate after L4-5 PLIF. However, the L2-3 and L4-5 segments have ligaments that are in a similar condition. Therefore, ASD is observed at both the cranial and caudal segments. Moreover, after L3-4 PLIF, the L4-5 segment is sandwiched between the fixed L3-4 segment and the rigid L5-S1 segment. As a result, motion is concentrated in the L4-5 segment, making that segment more vulnerable to degeneration than is the L2-3 segment.

Another factor is range of motion. Panjabi et al. ${ }^{16}$ reported that the main motion at the L4-5 level is significantly greater than that at the L2-3 level regarding flexion and extension moment loading. It is not difficult to assume that this large range of motion accelerates segment degeneration.

The final factor is that in the natural course of degeneration, the L4-5 segment more easily degenerates than the L2-3 segment does. Using the Pfirrmann classification,,$^{13} \mathrm{Oh}$ and Yoon $^{17}$ conducted a degeneration survey of 653 patients, and they observed grade IV or V degeneration in the L4-5 segment in $56.5 \%$ of those surveyed. In contrast, $23.3 \%$ of the patients had grade IV or V in the L2-3 segment. Moreover, Cheung et al. ${ }^{18}$ reported that in their cross-sectional study of changes shown on MRI of the lumbar spine, L4-5 was the second-most affected level next to the L5-S1 level, and that L2-3 was the secondleast affected level next to the L1-2 level. Thus, it is obvious that the L4-5 segment degenerates more easily than the L2-3 segment in the natural course of events. From these findings we can easily assume that degeneration of the L4-5 segment is more frequently observed compared with the L2-3 segment after L3-4 PLIF.

Our study had some limitations. First, it was a retrospective case series. Second, the number of study participants was not large enough to allow for generalization of our findings, because those who underwent L3-4 PLIF alone represented only $11.2 \%$ of all participants undergoing PLIF. Third, global alignment including spinopelvic parameters was not evaluated. There may be a relationship between ASD and these parameters. Fourth, we did not have control groups, because we believe that it is commonly recognized that the cranial adjacent segment often degenerates after L4-5 fusion surgery. We based that belief on many reports concerning ASD and lumbar fusion surgery. ${ }^{5-9}$ Future studies should be conducted to determine the risk of ASD in a larger number of patients with global alignment.

\section{Conclusions}

In our study, the incidences of R-ASD, S-ASD, and OASD were $48 \%, 15 \%$, and 9\%, respectively, after L3-4 PLIF for degenerative lumbar diseases. A caudal segment was involved in $67 \%$ of R-ASD cases, in $70 \%$ of S-ASD cases, and in $83 \%$ of O-ASD cases. Thus, we found that after L3-4 PLIF, ASD was more frequently observed at a caudal segment than at a cranial segment.

\section{References}

1. Dantas FL, Prandini MN, Ferreira MA. Comparison between posterior lumbar fusion with pedicle screws and posterior lumbar interbody fusion with pedicle screws in adult spondylolisthesis. Arq Neuropsiquiatr. 2007;65(3B):764-770.

2. Ha KY, Na KH, Shin JH, Kim KW. Comparison of posterolateral fusion with and without additional posterior lumbar interbody fusion for degenerative lumbar spondylolisthesis. $J$ Spinal Disord Tech. 2008;21(4):229-234.

3. Nakai S, Yoshizawa H, Kobayashi S. Long-term follow-up study of posterior lumbar interbody fusion. J Spinal Disord. 1999;12(4):293-299.

4. Okuda S, Miyauchi A, Oda T, et al. Surgical complications of posterior lumbar interbody fusion with total facetectomy in 251 patients. J Neurosurg Spine. 2006;4(4):304-309.

5. Chen BL, Wei FX, Ueyama K, et al. Adjacent segment degeneration after single-segment PLIF: the risk factor for degeneration and its impact on clinical outcomes. Eur Spine J. 2011;20(11):1946-1950.

6. Imagama S, Kawakami N, Matsubara Y, et al. Radiographic adjacent segment degeneration at 5 years after L4/5 posterior lumbar interbody fusion with pedicle screw instrumentation: evaluation by computed tomography and annual screening with magnetic resonance imaging. Clin Spine Surg. 2016;29(9):E442-E451.

7. Kaito T, Hosono N, Mukai Y, et al. Induction of early degeneration of the adjacent segment after posterior lumbar interbody fusion by excessive distraction of lumbar disc space. $J$ Neurosurg Spine. 2010;12(6):671-679.

8. Kim KH, Lee SH, Shim CS, et al. Adjacent segment disease after interbody fusion and pedicle screw fixations for isolated L4-L5 spondylolisthesis: a minimum five-year follow-up. Spine (Phila Pa 1976). 2010;35(6):625-634.

9. Okuda S, Iwasaki M, Miyauchi A, et al. Risk factors for adjacent segment degeneration after PLIF. Spine (Phila Pa 1976). 2004;29(14):1535-1540.

10. Aono H, Takenaka S, Nagamoto Y, et al. Fusion rate and clinical outcomes in two-level posterior lumbar interbody fusion. World Neurosurg. 2018;112:e473-e478.

11. Okuda S, Oda T, Miyauchi A, et al. Surgical outcomes of posterior lumbar interbody fusion in elderly patients. Surgical technique. J Bone Joint Surg Am. 2007;89(suppl 2 pt 2):310-320.

12. Nakashima H, Kawakami N, Tsuji T, et al. Adjacent segment disease after posterior lumbar interbody fusion: based on cases with a minimum of 10 years of follow-up. Spine (Phila Pa 1976). 2015;40(14):E831-E841.

13. Pfirrmann CW, Metzdorf A, Zanetti M, et al. Magnetic reso- 
nance classification of lumbar intervertebral disc degeneration. Spine (Phila Pa 1976). 2001;26(17):1873-1878.

14. Cloward RB. The treatment of ruptured lumbar intervertebral disc by vertebral body fusion. III. Method of use of banked bone. Ann Surg. 1952;136(6):987-992.

15. Okuda S, Nagamoto Y, Matsumoto T, et al. Adjacent segment disease after single segment posterior lumbar interbody fusion for degenerative spondylolisthesis: minimum 10 years follow-up. Spine (Phila Pa 1976). 2018;43(23):E1384-E1388.

16. Panjabi MM, Oxland TR, Yamamoto I, Crisco JJ. Mechanical behavior of the human lumbar and lumbosacral spine as shown by three-dimensional load-displacement curves. $J$ Bone Joint Surg Am. 1994;76(3):413-424.

17. $\mathrm{Oh} \mathrm{CH}$, Yoon SH. Whole spine disc degeneration survey according to the ages and sex using Pfirrmann disc degeneration grades. Korean J Spine. 2017;14(4):148-154.

18. Cheung KM, Karppinen J, Chan D, et al. Prevalence and pattern of lumbar magnetic resonance imaging changes in a population study of one thousand forty-three individuals. Spine (Phila Pa 1976). 2009;34(9):934-940.

\section{Disclosures}

The authors report no conflict of interest concerning the materials or methods used in this study or the findings specified in this paper.

\section{Author Contributions}

Conception and design: Aono. Acquisition of data: Aono, Tobimatsu, Nagamoto, Furuya, Yamashita, Ishiguro. Analysis and interpretation of data: Aono, Takenaka. Drafting the article: Aono. Critically revising the article: Aono, Yamashita. Reviewed submitted version of manuscript: Aono, Tobimatsu, Nagamoto, Furuya, Ishiguro, Iwasaki. Statistical analysis: Takenaka. Study supervision: Iwasaki.

\section{Correspondence}

Hiroyuki Aono: National Hospital Organization, Osaka National Hospital, Osaka, Japan. h-aono@umin.ac.jp. 